\title{
ANÁlisis DE CONTROLADORES POR REALIMENTACIÓN DE ESTADOS, CONTROL ÓPTIMO Y LÓGICA DIFUSA, PARA UN PÉNDULO DE FURUTA
}

\author{
Juan P. Ortiz ${ }^{1, *}$ y Manuel J. Reinoso ${ }^{2}$
}

\section{Resumen}

Este trabajo presenta el desarrollo y control de un péndulo invertido rotacional (péndulo de Furuta) mediante controladores en el espacio de estados, control óptimo y lógica difusa. Este tipo de sistemas son las aeronaves, vehículos espaciales, vehículos submarinos, barcos, satélites y robots, construidos por barras y uniones articuladas, pasivas y activas. Estos sistemas generalmente están desafiando a la gravedad y deben mantenerse en equilibrio, por lo tanto, las plantas de estos sistemas son no lineales y se hace necesario controlarlo mediante leyes de control no lineales; sin embargo, debido al aparecimiento de la lógica difusa, este tipo de control tiene una gran ventaja que radica en que no es necesario saber con exactitud las características de la planta; el control puede ser adaptado para sistemas SISO y MIMO; la clave del control difuso radica especialmente en el conocimiento que debe tener el experto para plantear los rangos de las funciones de inferencia y su tipo de funciones, además, plantear el método de inferencia de la máquina de inferencia; por lo tanto, para este tipo de control se debe hacer varias pruebas hasta conseguir los objetivos planteados en el sistema de control.

Palabras clave: Péndulo Furuta, espacio de estados, control óptimo, lógica difusa, simple input simple output (SISO), multiple input multiple output (MIMO), método de inferencia.

\section{Abstract}

This paper presents the development and control of a rotational inverted pendulum (Furuta pendulum) by controllers in the state space, optimal control and fuzzy logic. Such systems are aircraft, spacecraft, underwater vehicles, ships, satellites and robots, built by rods and articulated joints, passive and active. These systems generally are defying gravity to stay in balance so plants such systems are nonlinear and will need to be monitored by nonlinear control laws however due to the appearance of fuzzy logic, this control has great advantage is that it is not necessary to know exactly the characteristics of the plant and control systems can be adapted to Simple Simple Input Output (SISO) or Multiple Input Multiple Output (MIMO), the key lies especially fuzzy control in the knowledge that you need to ask the expert ranges inference functions and features such well raise the inference method of inference engine, so for this type of control should be done several tests to get the goals set in the control system.

Keywords: Furuta pendulum, state space, optimal control, fuzzy logic, single input single output (SISO), multiple input multiple output (MIMO), inference method.

\footnotetext{
1,* Ingeniero electrónico, egresado de la Maestría en Control y Automatización Industrial, Universidad Politécnica Salesiana, sede Cuenca. Autor para correspondencia paul_ortiz02@hotmail.com

${ }^{2}$ Ingeniero electrónico, egresado de la Maestría en Control y Automatización Industrial, Universidad Politécnica Salesiana, sede Cuenca. Autor para correspondencia manuelrja@hotmail.com

Recibido: 01-10-2012, Aprobado tras revisión: 11-06- 2013.

Forma sugerida de citación: Ortiz J. y Reinoso, J. (2013). "Análisis de controladores por realimentación de estados, control óptimo y lógica difusa, para un péndulo de Furuta ". INGEnIUs. N. ${ }^{\circ}$, (Enero-Junio). pp. 15-22. ISSN: 1390-650X.
} 


\section{Introducción}

Dentro de los sistemas de péndulos invertidos, un ejemplo muy utilizado y profundamente estudiado es el péndulo encima de un carro móvil, se utiliza para el análisis experimental de técnicas de control. El inconveniente con este sistema se encuentra en que el recorrido del carro se encuentra acotado, lo que limita las maniobras de control. Para eliminar dicha limitación se reemplaza la trayectoria lineal del carro por una circular, dando origen al sistema péndulo de Furuta, dicho péndulo fue creado por el Dr. K. Furuta del Instituto de Tecnología de Tokio, Japón. Es un sistema MISO subactuado de dos grados de libertad rotacionales llamados brazo y péndulo.

El movimiento del brazo se realiza en un plano horizontal girando alrededor de un eje al plano, el péndulo se encuentra ubicado en un extremo del brazo y su eje giro es colineal al eje axial del brazo y su movimiento se realiza en un plano perpendicular al de este último [1].

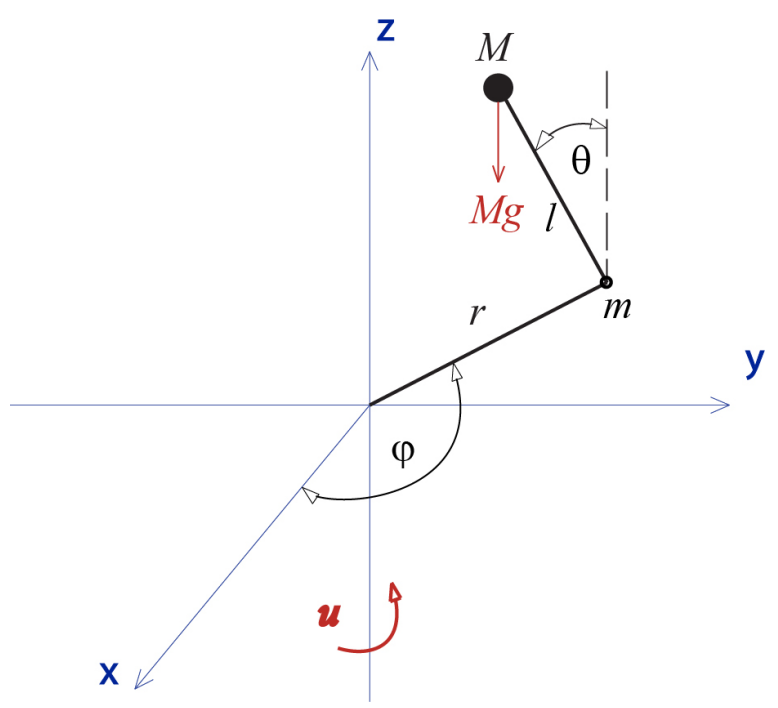

Figura 1. Esquema del péndulo de Furuta.

Se presenta en la Figura 1 el esquema típico de un péndulo de Furuta donde se muestra el actuador y los dos movimientos que se dan para tratar de llevarlo al equilibrio.

Está formado por dos cuerpos inerciales que se encuentran unidos entre sí: un eslabón central o brazo con momento de inercia $J$ de dimensión $r$ y masa homogéneamente distribuida $m$. El péndulo de longitud $l_{p}$ y masa homogéneamente distribuida en línea $m_{p}$. El ángulo del péndulo, $\theta$, ha sido definido como cero en la posición vertical arriba, y positivo, cuando el péndulo se mueve en la dirección de las manecillas del reloj.

El ángulo del brazo, $\phi$, se ha definido positivo cuando el brazo se mueve en la dirección contraria a las manecillas del reloj. En la parte central del brazo de longitud $r$, se encuentra acoplado un motor de DC el mismo que producirá la señal $u$ de control para producir el estado estable es decir, que el péndulo permanezca en equilibrio.

\subsection{Modelado no lineal para el sistema}

El modelo de un péndulo de Furuta se lo realiza en base a la teoría de Euler-Lagrange [2], mediante la ubicación de un punto en el espacio que genera la ubicación final del péndulo y las distancias en función de los ángulos de desplazamiento y conjuntamente con las ecuaciones de movimiento se generan dos ecuaciones de energía la cual mediante el langraniano se obtienen las ecuaciones 1 y 2.

$$
\begin{gathered}
\left(J_{p}+M l^{2}\right)\left(\theta-\phi^{2} \sin (\ddot{\theta}) \cos (\theta)\right)+\ldots \\
\ldots+M r l \ddot{\phi} \cos (\theta)-g l\left(M+\frac{m}{2}\right) \sin (\theta) \\
M r l \ddot{\theta} \cos (\theta)-M r l \dot{\theta} \sin (\theta)+\ldots \\
\ldots+2\left(J_{p}+m l^{2}\right)(\dot{\theta} \dot{\phi} \sin (\theta) \cos (\theta))+\ldots \\
\ldots+g l\left[J+m r+M r+\left(J_{p}+m l\right) \sin ^{2}(\theta)\right] \ddot{\phi}=u
\end{gathered}
$$

Estas son las ecuaciones dinámicas que rigen al sistema, observándose que el sistema es no lineal por ser cuadrática y tener funciones trigonométricas.

A continuación se muestra el valor de los constantes utilizadas para la simulación del sistema del péndulo de Furuta:

$$
\begin{gathered}
l=0,413[\mathrm{~m}] \\
M=0,413[\mathrm{~kg}] \\
J_{p}=9 \times 10^{-4}\left[\mathrm{~kg} \cdot \mathrm{m}^{2}\right] \\
r=0,235[\mathrm{~m}] \\
m=0,02[\mathrm{~kg}] \\
J=0,05\left[\mathrm{~kg} \cdot \mathrm{m}^{2}\right]
\end{gathered}
$$

\subsection{Linelización del sistema}

Se trata de eliminar las no linealidades del sistema del péndulo de Furuta [3], y esto se realiza linealizando alrededor del punto de equilibrio cuando las cuatro variables de estado estén muy cercanamente a $x=\left[\begin{array}{llll}0 & 0 & 0 & 0\end{array}\right]$, por lo tanto sabiendo que las variables de estado son:

$$
x=\left[\begin{array}{llll}
\theta & \dot{\theta} & \phi & \dot{\phi}
\end{array}\right]
$$

El sistema linealizado y en variables de estado se tiene en la ecuación 3. 
$\dot{x}=A x+B u=\left[\begin{array}{cccc}0 & 1 & 0 & 0 \\ \frac{b d}{a b-c^{2}} & 0 & 0 & 0 \\ 0 & 0 & 0 & 1 \\ \frac{-c d}{a b-c^{2}} & 0 & 0 & 0\end{array}\right] x+\left[\begin{array}{c}0 \\ \frac{-c g}{a b-c^{2}} \\ 0 \\ \frac{a g}{a b-c^{2}}\end{array}\right] u$

Donde las constantes son:

$$
\begin{array}{cc}
a=J_{p}+M l^{2} & b=J+M r^{2}+m r^{2} \\
c=M r l & d=l g\left(M+\frac{m}{2}\right)
\end{array}
$$

Reemplazando los valores de las constantes en la ecuación 3 se obtiene la ecuación 5 .

$$
\begin{gathered}
\dot{x}=A x+B u=\left[\begin{array}{cccc}
0 & 1 & 0 & 0 \\
31,3167 & 0 & 0 & 0 \\
0 & 0 & 0 & 1 \\
-0,5854 & 0 & 0 & 0
\end{array}\right] x+\ldots \\
\ldots+\left[\begin{array}{c}
0 \\
-71,2340 \\
0 \\
191,2458
\end{array}\right] u
\end{gathered}
$$

Tomando un tiempo de muestreo de $T_{s}=0.05 \mathrm{~s} \mathrm{y}$ utilizando MATLAB ${ }^{\circledR}$ para tomar la discretización de las matrices se tiene:

Afd $=$

$$
\begin{array}{cccc}
1,0016 & 0,0100 & 0 & 0 \\
0,3133 & 1,0016 & 0 & 0 \\
-0,0000 & -0,0000 & 1,0000 & 0,0100 \\
-0,0059 & -0,0000 & 0 & 1,0000
\end{array}
$$

$\operatorname{Bfd}=$

$$
\begin{gathered}
-0,0036 \\
-0,7127 \\
0,0096 \\
1,9125
\end{gathered}
$$

$\operatorname{Cfd}=$

$\begin{array}{llll}1 & 0 & 0 & 0 \\ 0 & 1 & 0 & 0 \\ 0 & 0 & 1 & 0 \\ 0 & 0 & 0 & 1\end{array}$

$\operatorname{Hfd}=$

$$
0
$$

\section{Diseño de controladores para el péndulo de Furuta}

En la presente sección se plantea el diseño de tres controladores para el control del péndulo de Furuta, el primer controlador será de realimentación de estados, el segundo basado en control óptimo y el tercero por medio de lógica difusa.

\subsection{Control de realimentación de estados}

Se plantea una ley de control para sistemas lineales en espacio de estados que es la realimentación de los mismos.

$$
u(k)=-K x(k)
$$

Ahora se halla $K$ mediante ubicación de polo de bessel con $T=0.5 \mathrm{~s}$ para el tiempo de estabilización, a continuación se muestra el código implementado en MATLAB ${ }^{\circledR}$ para hallar la ganancia $K$ de realimentación de estados:

Código 1. Estabilización de polos tipo Bessel.

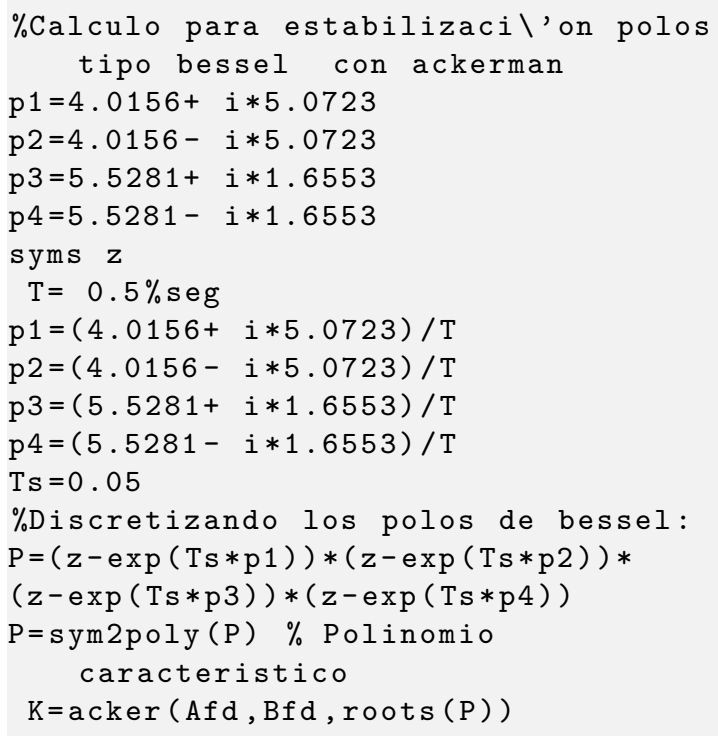

El vector de ganancias $K$ resultante es:

$$
K=\left[\begin{array}{llll}
-23,3113 & 3,7306 & -4,5396 & 1,1679
\end{array}\right]
$$

La simulación se realiza mediante Simulink ${ }^{\circledR}$, el diagrama de bloques se muestra en la Figura 2.

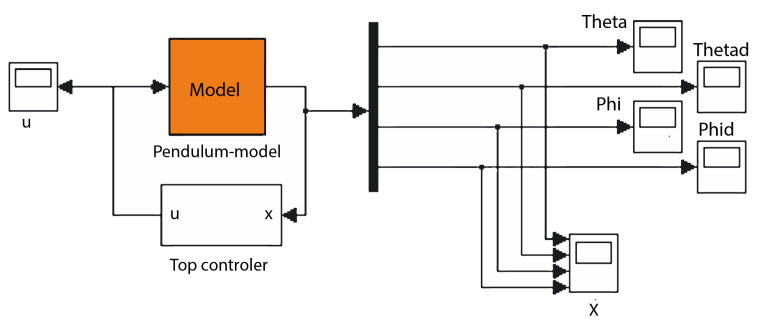

Figura 2. Diagrama en Simulink ${ }^{\circledR}$ para la simulación del péndulo de Furuta con un vector $K$ en realimentación de estados.

Como se puede observar en la Figura 3, ante ningún polo de Bessel el sistema da una correcta respuesta de estabilidad por lo tanto el vector $K$ de realimentación 
de estados saca al sistema rápidamente de la zona de estabilidad, se concluye que este tipo de control con reubicación de polos no es válida para la estabilidad de este sistema.

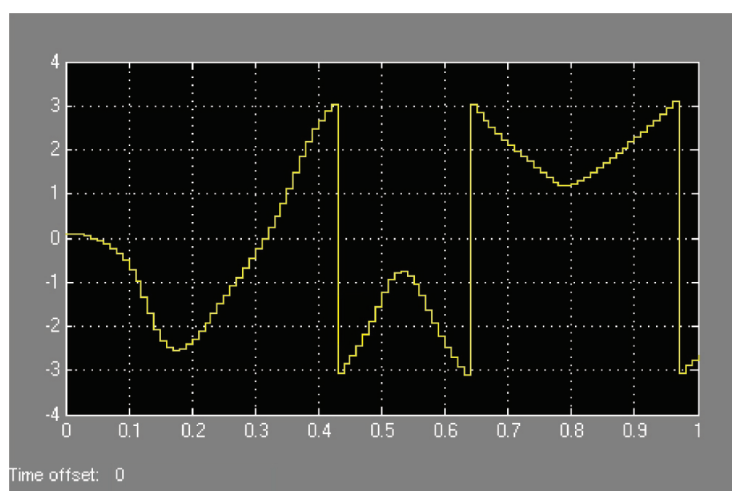

Figura 3. Ángulo $\theta$ (rad) de respuesta con ganancia $K$ por ubicación de polos de bessel con un tiempo de muestreo $T_{s}=0.5 \mathrm{~s}$

\subsection{Control óptimo mediante realimentación de estados}

Al observar que el sistema no se estabiliza con la reubicación de polos de Bessel, ahora se plantea un $K$ óptimo tratando de minimizar la función de coste del error del sistema regulador que llevaría a la estabilidad del sistema, donde dada una condición inicial $x(0)$ se trata de hallar un vector de control permitido $u(k)$ que transfiera al estado a la región deseada del espacio de estados que para nuestro sistema será $x(0)=\left[\begin{array}{llll}0 & 0 & 0 & 0\end{array}\right]$ y para el cual lo que se minimiza es el índice de desempeño [4].

Elementos claves para la implementación de control óptimo:

- La forma en la que se predice lo que pueda pasar (modelo).

- La herramienta que permita valorar estas mediciones (medición).

- El instrumento para implementar las actividades (control).

A continuación se propone el siguiente regulador lineal cuadrático (LQR), técnica de control lineal por realimentación del estado para estabilizar localmente el punto de equilibrio.

Para obtener la matriz de ganancia de realimentación de estado $K$ se utiliza el control óptimo LQR. Este regulador calcula la matriz de ganancia óptima tal que la ley de realimentación de estado $u(k)=-K x(k)$ minimiza la función de costo de la ecuación 6 .

$$
J=\sum x^{T} Q x+u^{T} R u
$$

Los parámetros de diseño de este controlador son las matrices de peso $Q$ y $R$. $Q$ se utiliza como pesos de penalización para los estados y $R$ para penalizar la señal de control. Ahora mediante MATLAB ${ }^{\circledR}$ se crea la siguiente matriz $Q$ y $R$ donde:

$$
Q=\left[\begin{array}{cccc}
100 & 0 & 0 & 0 \\
0 & 1 & 0 & 0 \\
0 & 0 & 10 & 0 \\
0 & 0 & 0 & 1
\end{array}\right] \quad R=10
$$

Código para hallar el $K$ óptimo mediante comandos de MATLAB ${ }^{\circledR}$ :

Código 2. $K$ óptimo.

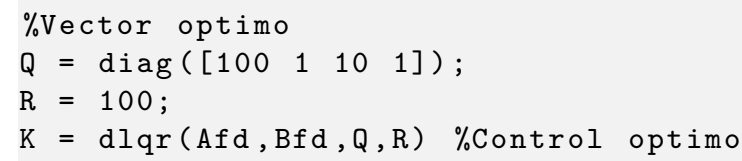

Dónde $K$

$$
K=\left[\begin{array}{llll}
-5,3983 & -0,95946 & -0,2663 & -0,1968
\end{array}\right]
$$

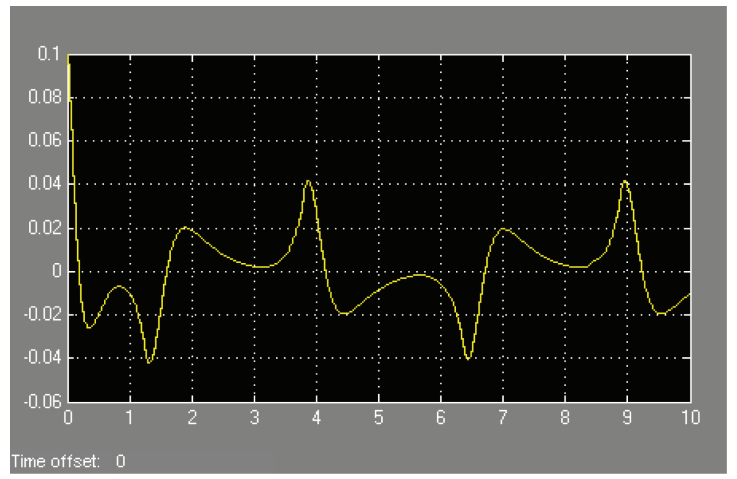

Figura 4. Ángulo $\theta(\mathrm{rad})$ de respuesta ante la entrada $K$ óptimo.

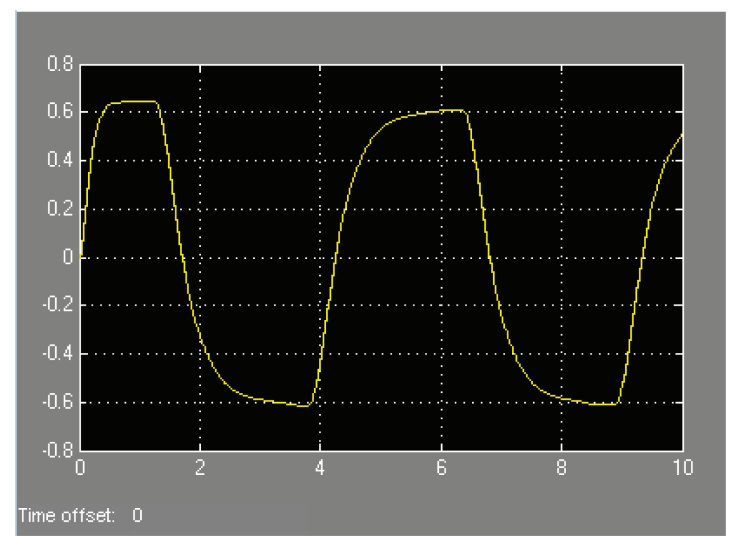

Figura 5. Ángulo $\phi(\mathrm{rad})$ de respuesta ante la entrada $K$ óptimo. 
En las Figuras 4 y 5 se observa claramente que el sistema no se estabiliza totalmente en el ángulo $\theta$, tiene una oscilación de unos pocos grados alrededor del punto $0^{\circ}$, sin embargo, el ángulo $\phi$ hace una serie de oscilaciones, donde se concluye que dicho vector $K$ óptimo estabiliza en parte el sistema pero no en el punto $x=\left[\begin{array}{llll}0 & 0 & 0 & 0\end{array}\right]$.

Ahora se va a variar la matriz $Q$ con el objetivo de evitar la oscilación excesiva alrededor del ángulo $\theta$. Se plantea la matriz $Q$ como sigue, tratando de penalizar a toda costa la tercera variable que oscila el ángulo $\phi$, por lo que en esa variable ponemos un valor muy cercanamente a cero:

$$
Q=\left[\begin{array}{cccc}
100 & 0 & 0 & 0 \\
0 & 1 & 0 & 0 \\
0 & 0 & 10 \times \exp ^{-10} & 0 \\
0 & 0 & 0 & 10
\end{array}\right]
$$

Mantenemos el mismo valor para $R$ y hallamos nuevamente el vector $K$ óptimo que para este caso resulta:

$$
x=\left[\begin{array}{llll}
-7,5343 & -1,3465 & -0,0000 & -0,2216
\end{array}\right] .
$$

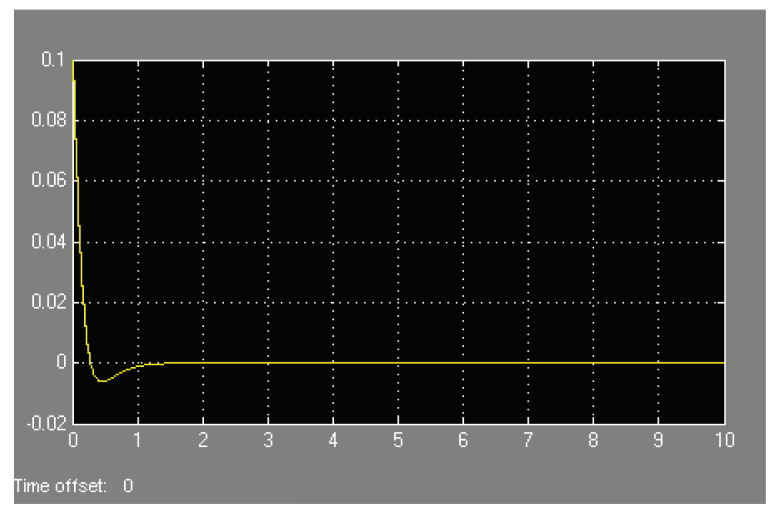

Figura 6. Ángulo $\theta$ (rad) de respuesta ante la entrada $K$ óptimo.

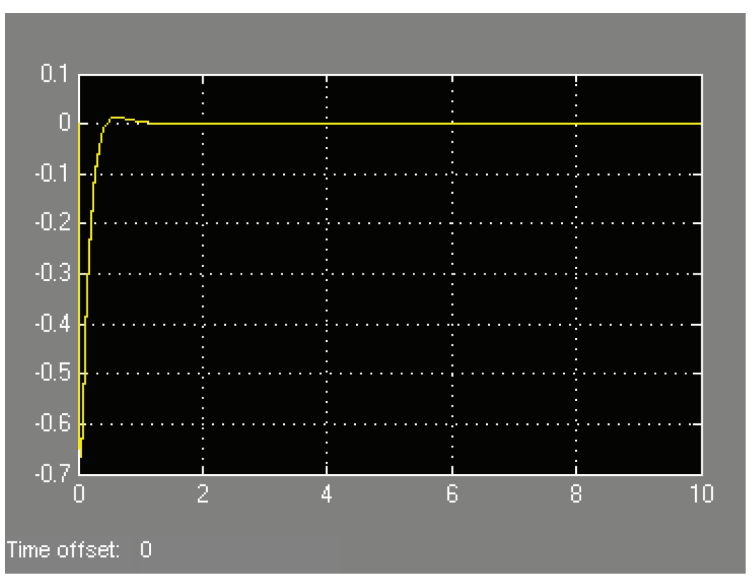

Figura 7. Velocidad de cambio del ángulo $\theta$.
$\mathrm{Al}$ observar las Figuras 6, 7, 8 y 9 se tiene muy claro que el control óptimo hace su mejor esfuerzo por estabilizar el ángulo $\theta$ del péndulo (Figura 6) pero el brazo permanece girando ya que jamás se detiene, eso explica que el ángulo $\phi$ de la Figura 8 crece indefinidamente en un solo sentido a velocidad constante, por lo que el vector de ganancias $K$ óptimo no lleva completamente al punto de equilibrio $x=\left[\begin{array}{llll}0 & 0 & 0 & 0\end{array}\right]$, esto hace necesario un nuevo control.

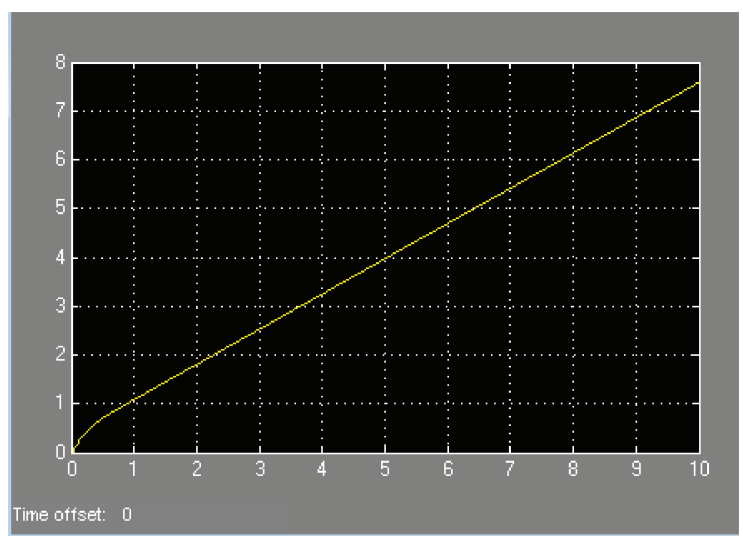

Figura 8. Ángulo $\phi$ ante el $K$ óptimo

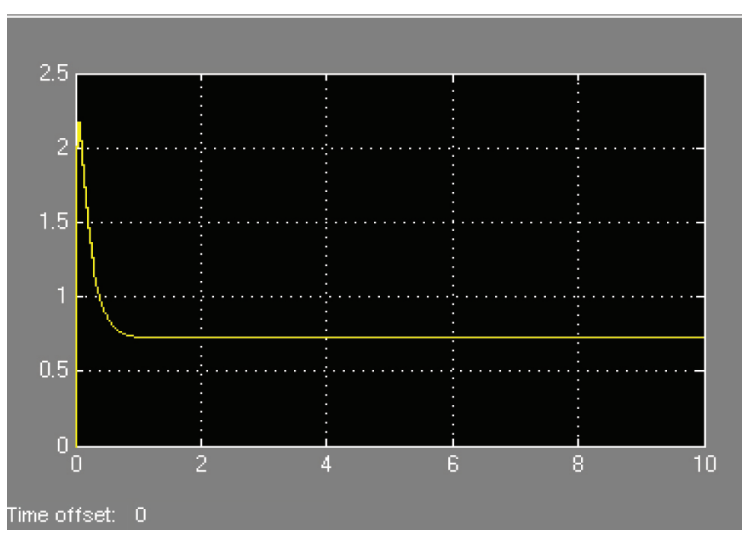

Figura 9. Velocidad de cambio del ángulo $\phi$.

\subsection{Control difuso}

Este documento no pretende estudiar o presentar los conocimientos básicos de diseño de controladores difusos, por el contrario se espera que el lector posee dichos conocimientos o de no ser el caso tendrá que realizar un estudio previo para esta sección (ver [2]).

El sistema de péndulo de Furuta como ya se analizó anteriormente, posee cuatro entradas mostradas en la Figura 10 que serán el objeto de control.

El controlador será diseñado mediante la siguiente herramienta de MATLAB ${ }^{\circledR}$ :

$>$ fuzzy 
La misma que presenta un entorno de desarrollo amigable para el planteamiento de las condiciones del controlador, tanto en funciones de membresía, método de fuzificación, reglas, implicación difusa y defusificación de la salida que en este caso es la señal de control entregada a la planta. La Figura 10 presenta las variables de entrada y salida del sistema.

\section{FIS Variables}
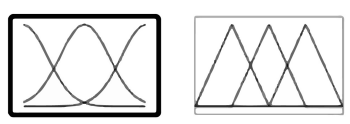

teta

u

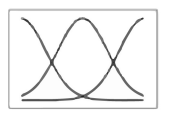

tetad

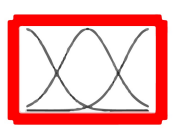

phi

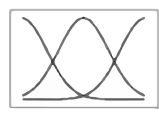

phid

Figura 10. Variables de entrada y salida.

Los rangos de entrada para la variable $\theta$ se muestran en la Figura 11. Las funciones de pertenencia triangulares correspondientes a la variable de entrada $\theta$ son:

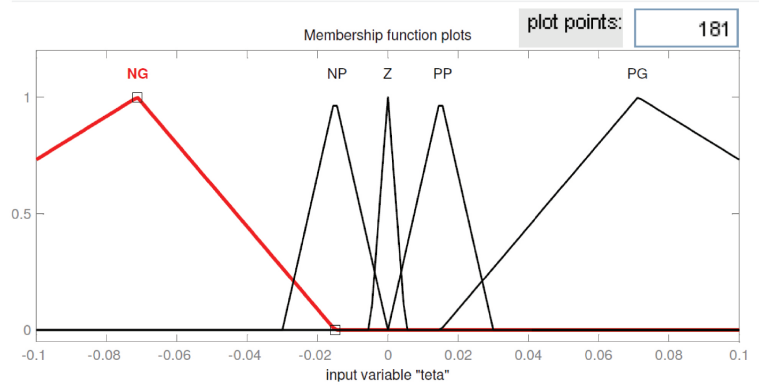

Figura 11. Variable de entrada $\theta$.

$$
\begin{array}{cl}
\mathrm{NG} & \rightarrow \text { Negativo grande } \\
\mathrm{NP} & \rightarrow \text { Negativo pequeño } \\
\mathrm{Z} & \rightarrow \text { Cero } \\
\mathrm{PP} & \rightarrow \text { Positivo pequeño } \\
\mathrm{PG} & \rightarrow \text { Positivo grande }
\end{array}
$$

Para la variable $\theta_{d}$ que hace referencia a la velocidad angular de $\theta$ se muestra en la Figura 12.

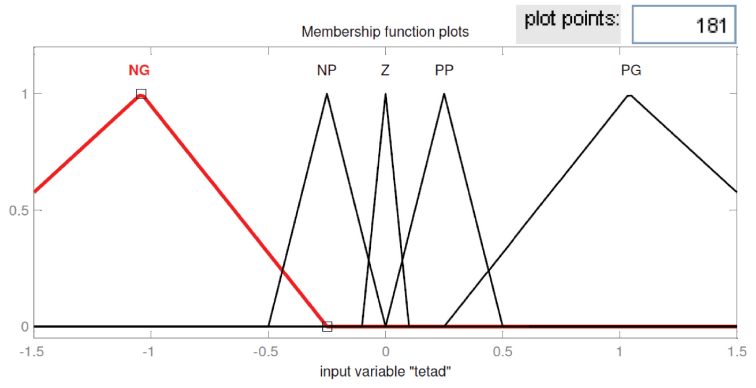

Figura 12. Variable de entrada $\theta_{d}$.

De igual forma las funciones de pertenencia triangulares correspondientes a la Figura 12, velocidad angular son:

$$
\begin{aligned}
\mathrm{NG} & \rightarrow \text { Negativo grande } \\
\mathrm{NP} & \rightarrow \text { Negativo pequeño } \\
\mathrm{Z} & \rightarrow \text { Cero } \\
\mathrm{PP} & \rightarrow \text { Positivo pequeño }
\end{aligned}
$$

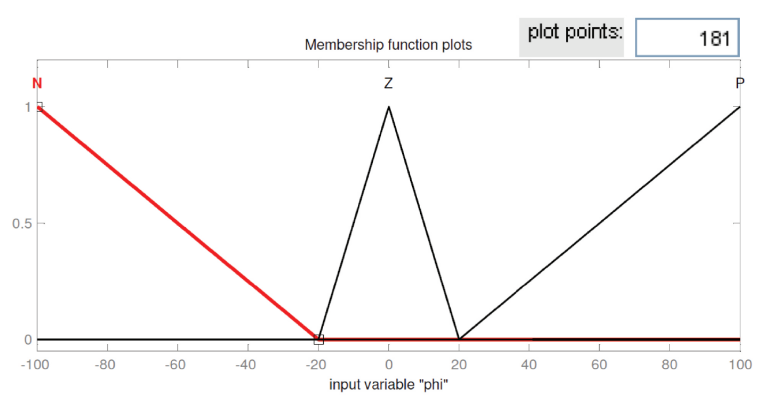

Figura 13. Variable de entrada $\phi$.

NG $\rightarrow$ Positivo grande

Para $\phi$ de la Figura 13, las funciones de pertenencia están definidas como sigue:

$$
\begin{aligned}
& \mathrm{N} \rightarrow \text { Negativo } \\
& \mathrm{Z} \rightarrow \text { Cero } \\
& \mathrm{PP} \rightarrow \text { Positivo }
\end{aligned}
$$

La velocidad angular de $\phi$ nombrada $\phi_{d}$ se muestra en la Figura 14. Para la señal de control de la Figura 15 se seleccionó funciones de pertenencia triangulares con las variables lingüísticas siguientes:

$$
\begin{aligned}
\mathrm{NG} & \rightarrow \text { Negativo grande } \\
\mathrm{NP} & \rightarrow \text { Negativo pequeño } \\
\mathrm{Z} & \rightarrow \text { Cero } \\
\mathrm{PP} & \rightarrow \text { Positivo }
\end{aligned}
$$




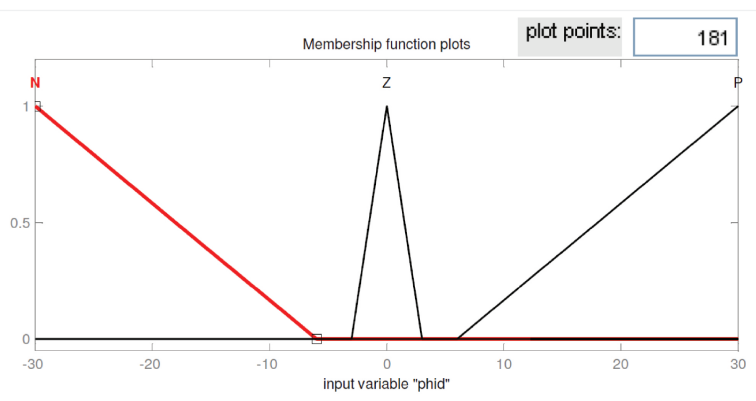

Figura 14. Variable de entrada $\phi_{d}$.

$\mathrm{PG} \rightarrow$ Positivo grande

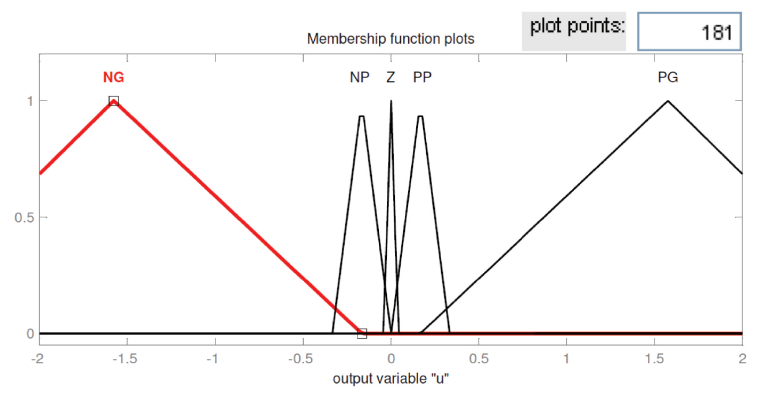

Figura 15. Variable de salida $u$.

Las reglas de implicación usadas en el controlador se muestran en la Tabla 1, usando la máquina de inferencia de Mandami y el método del centroide para la defuzificación, se plantearon las siguientes reglas:

Tabla 1. Reglas de fuzificación.

\begin{tabular}{rccccc}
\hline \multirow{2}{*}{ Vel. ang. } & \multicolumn{5}{c}{ Ángulo } \\
\cline { 2 - 6 } & NG & NP & Z & PP & PG \\
\hline NG & NG & NG & NG & Z & PG \\
NP & NG & NP & NP & PP & PG \\
Z & NG & NP & Z & PP & PG \\
PP & NG & NP & PP & PP & PG \\
PG & NG & Z & PG & PG & PG \\
\hline
\end{tabular}

En la Tabla 1 se observar la base de conocimiento simétrica, la misma que se puede ampliar a las variables $\theta_{d}, \phi \mathrm{y} \phi_{d}$.

Es de gran ayuda en el desarrollo del controlador verificar que las reglas de fuzificación cumplan con los criterios establecidos. MATLAB ${ }^{\circledR}$ proporciona una interfaz de usuario llamado Rule Viewer que permite evaluar las reglas de fuzificación.

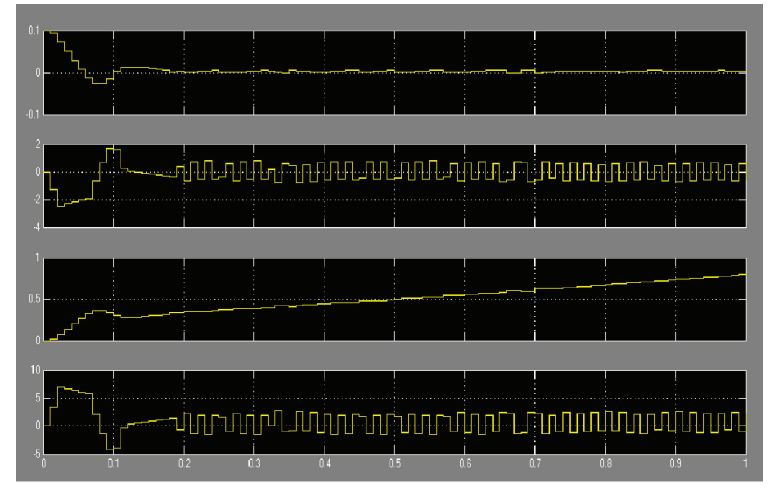

Figura 16. Resultados de los estados del sistema.

En la Figura 16 se encuentra las respuestas de las variables $\theta, \theta_{d}, \phi$ y $\phi_{d}$, en donde se muestra que el sistema posee estabilidad.

\subsection{Simulación del controlador difuso}

El diagrama de bloques usado para la simulación se indica en la Figura 17, en donde las entradas del controlador son las variables de estado y la salida es la señal de control.

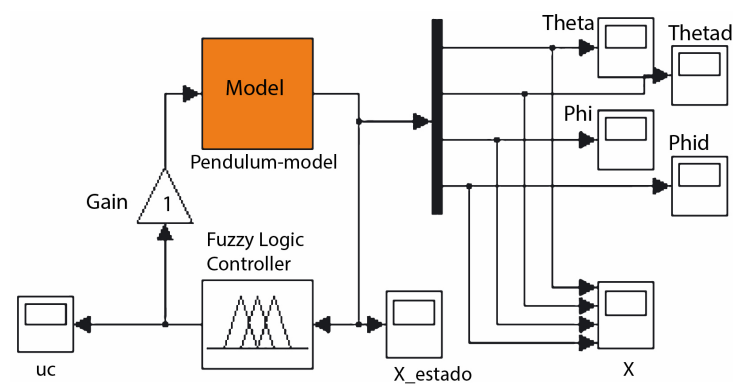

Figura 17. Diagrama de bloques del controlador difuso.

El modelo del péndulo Furuta mostrado anteriormente estaba basado en el desarrollo de:

Código 3. Créditos del código.

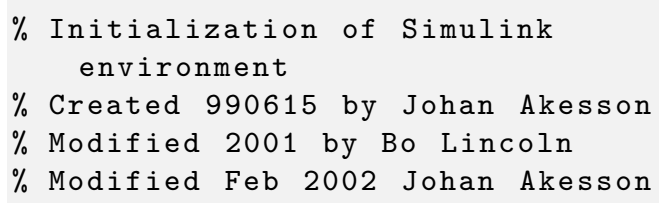

\section{Resultados y discusión}

Analizando el desempeño de los distintos controladores, se puede mencionar que el controlador de realimentación de estados no consigue la estabilidad del sistema por lo que se descarta en primera instancia; por otro lado, el controlador óptimo que trabaja minimizando 
la función de costo de la ecuación 7, consigue la estabilidad del sistema en un tiempo de 1 segundo para los parámetros iniciales $x_{0}=\left[\begin{array}{llll}0 & 0 & 0 & 0\end{array}\right]$.

$\mathrm{Al}$ estudiar el desempeño del controlador difuso mostrado en la Figura 16 se puede apreciar su influencia en la variables de estado, obteniendo un tiempo de establecimiento mucho menor para los parámetros iniciales $x_{0}=\left[\begin{array}{llll}0 & 0 & 0 & 0\end{array}\right]$, este tiempo fue de $0.15 \mathrm{~s}$.

El sistema con el controlador difuso mantiene la estabilidad a lo largo de todo tiempo $t$, teniéndose en cuenta que las reglas definidas estabilizan el ángulo $\theta$, más no el ángulo $\phi$ que permanece con velocidad constante. De los tres controladores estudiados en el presente trabajo se establece al controlador difuso como el que proporciona el mejor desempeño medido por su pequeño tiempo de establecimiento.

El completar el control para $\phi$ implica tener un conocimiento amplio de todo el comportamiento del péndulo y sus influencias entre sí, elevando la complejidad del desarrollo sin ser la finalidad del presente trabajo.

El principal reto para el desarrollo del controlador difuso sin duda es el establecer correctamente los limites y las funciones de pertenencia de las variables de entrada como de la salida. Es posible de ser necesario establecer pesos a las reglas para que tengan mayor o menor importancia.

\section{Conclusiones}

El control óptimo con el algoritmo LQR específicamente funciona mejor que el vector de retroalimentación de estados por reubicación de polos, sin embargo, no puede llevarlo al punto de equilibrio total donde todas las variables son nulas, esto se debe básicamente a que controla óptimamente solo al ángulo $\theta$ y no al ángulo $\phi$ por lo que será necesario la implementación adicional de una etapa de seguimiento de trayectoria; por ejemplo un controlador adaptivo difuso, para conseguir un sistema seguidor-controlador, donde la variable a seguir sea el ángulo $\phi$ y a controlar sea la variable $\theta$.

Para obtener un óptimo desempeño del controlador difuso es necesario conocer a fondo los valores que tiene la planta al igual que las variables de estado, ya que con estas medidas se puede aplicar las funciones de pertenencia de una mejor manera, pero sin duda alguna el éxito de estos controladores se ha debido básicamente a la prueba de muchas maneras y posibilidades en las que se pueden combinar las entradas para dar una salida acorde a la realidad que permita que el sistema se estabilice.

Es un trabajo largo establecer los parámetros de las funciones de pertenencia y establecer correctamente las reglas difusas para el funcionamiento del controlador. Como recomendación es necesario el levantamiento de información para la caracterización de la planta, en este caso establecer la magnitud adecuada del torque aplicado en la planta.

\section{Referencias}

[1] K. M. Passino and S. Yurkovich, Fuzzy control. Citeseer, 1998.

[2] M. Gfvert, "Derivation of furuta pendulum dynamics," Department of Automatic Control, Lund Institute of Technology, Lund, Sweden, Tech. Rep., 1998.

[3] X.-Y. Lu, "Control of nonlinear non-minimum phase systems using dynamic sliding mode," International Journal of Systems Science, vol. 30, no. 2, pp. 183-198, 1999.

[4] I. Minchla and R. Matban, "Nonlinear control of the furuta pendulum," ITESM, México, Tech. Rep., 2009.

[5] R. Olfati-Saber, "Fixed point controllers and stabilization of the cart-pole system and the rotating pendulum," in Proceedings of the 38th IEEE Conference on Decision and Control, vol. 2. IEEE, 1999, pp. 1174-1181.

[6] B. C. Kuo and M. F. Golnaraghi, Automatic control systems. John Wiley \& Sons New York, 2003, vol. 4 .

[7] R. C. Dorf and R. H. Bishop, Modern control systems. Pearson, 2011.

[8] K. Ogata, Ingeniería de control moderna. Pearson Educación, 2003.

[9] I. Fantoni and R. Lozano, Non-linear control for underactuated mechanical systems. Springer, 2001.

[10] J.-J. E. Slotine, W. Li et al., Applied nonlinear control. Prentice hall New Jersey, 1991, vol. 1, no. 1. 